\title{
Proximal Tibiofibular Synostosis and its Clinical Significance: A Case Report
}

\author{
Tiwari $S^{1}$, Roopashree $R^{2}$, Padmavathi $G^{3}$, Sangeeta $M^{4}$ \\ ${ }^{1}$ Dr Suman Tiwari, Assistant Professor, ${ }^{2}$ Dr Roopashree Ramakrishna, Associate Professor, ${ }^{3}$ Dr. G. Padmavathi, ${ }^{4}$ Dr M \\ Sangeeta, Professor and HOD. All are affiliated with Department of Anatomy, MVJ Medical College and Research Hospital, \\ Bangalore, India.
}

Address for correspondence: Dr Suman Tiwari, E-mail: sumanravi80@gmail.com

\begin{abstract}
Proximal tibiofibular synostosis is rare and is either congenital or associated with trauma. Very few cases have been reported in the literature. During routine examination of various dried and processed bones in the Department of Anatomy, it was found that the upper end of tibia and fibula were fused by an abnormal piece of bone. The length of tibia and fibula and the length and thickness of ossified part of bone measured. The position of proximal tibiofibular joint, interosseous distance and curvature of tibia and fibula studied. The present case is useful for anatomists, radiologists and Orthopaedic surgeons who are dealing with this region.
\end{abstract}

Keywords: Proximal, Tibiofibular, Synostosis

\section{Introduction}

Tibio-fibular synostosis is an unusual anatomic finding discovered incidentally on routine radiographs. A tibiofibular synostosis may be proximal, middle or distal in position. Proximal tibiofibular synostosis is rare. Synostosis at this site may be congenital, idiopathic, secondary to osteochondroma, secondary to multiple hereditary exostoses or caused by trauma with or without fracture [1]. An osteology specimen of proximal tibiofibular synostosis and its clinical significance is discussed.

\section{Case report}

\section{Description of specimen}

An osteology specimen of tibia and fibula with proximal tibiofibular synostosis is described. The proximal end of the tibia and fibula were fused by an abnormal piece of bone. An extra piece of bone was found arising from upper end of shaft of tibia. The specimen belongs to the left side. The measurements of the specimen were taken with the help of a sliding vernier caliper and flexible steel tape. The various measurements of the specimen are as follows.

\section{Measurements}

- Length of tibia- $34.8 \mathrm{~cm}$

- Length of fibula- $32.8 \mathrm{~cm}$

- Length of ossified part- $4.5 \mathrm{~cm}$
- Thickness of ossified part- $1.5 \mathrm{~cm}$

- Interosseous distance- $1.6 \mathrm{~cm}$

- The rest of the shaft of the tibia and fibula were normal and did not show any deformity. The Proximal and distal tibio-fibular joint were normal in position. There was no evidence of fracture but callus formation is seen at the proximal end of tibia and fibula.

\section{Discussion}

Union between adjacent bones or parts of a single bone made of osseous material; such as ossified connecting cartilage or fibrous tissue constitutes a synostosis [2]. Rahm described the first case of synostosis in a 43 year old patient [3]. Proximal synostosis is usually congenital, while the distal joint involvement is mostly acquired [4]. Radiologically, O’Dwyer has classified it into 3 types [5]

Type I has a straight fibula with synostosis occurring proximally, the cause of which is trauma.

Type II has a fibula of normal length with mild bowing and widening of interosseous distance in proximal half

Type III has synostosis at a more distal level than type II and marked bowing of fibula which occurs throughout its length with increased interosseous distance occurring into the distal half.

Takai et al. [6] proposed that the classification of O'Dwyer should be extended with a type-4 synostosis, because no deformity, bowing or length discrepancy of the fibula was present in their case. They suggested that 
the type- 4 synostosis occurred after closure of the growth plate, with repeated mechanical stress to the tibiofibular joint as a possible cause. The

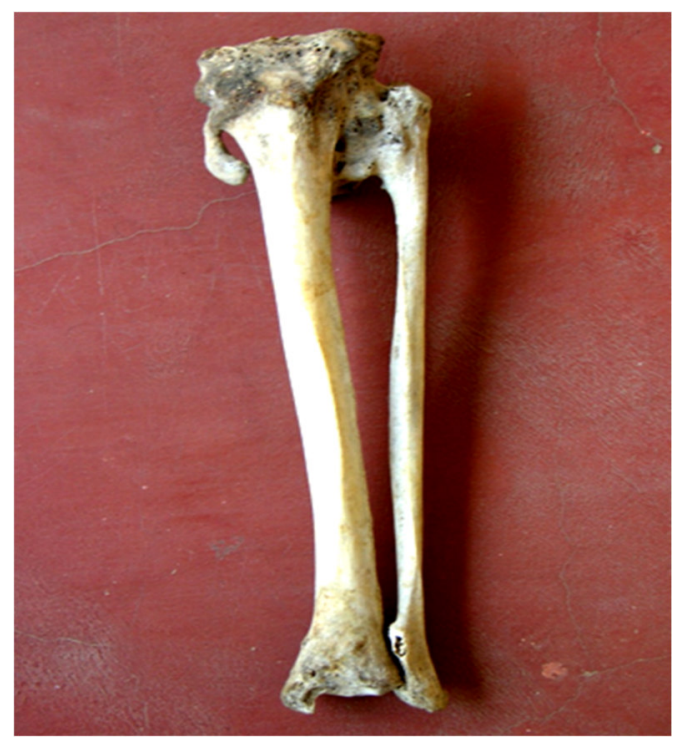

Fig 1: Anterior view of left tibia and fibula showing synostosis

The congenital tibiofibular synostosis is due to persistence of embryonal development stage of incompletely separated of the cartilage common to tibia and fibula during development of these bones [8].

Gamble et al [9] and O'Dwyer et al [5] stated that whenever the synostosis is present from birth or occurs before the closure of the proximal tibial growth plate, it is often symptomatic and may be associated with other growth deformities. The absence of any growth abnormalities indicates that the synostosis occurred after physeal closure. In the present case, the fibula is of normal length and did not show any deformity. Also, the proximal tibiofibular joint is normal in position. This suggests that synostosis developed in adult life that is, after the closure of epiphysis. The present case also can be classified under type 4 synostosis as suggested by takai and nishikawa.

Proximal Tibiofibular synostosis can be asymptomatic or can present with different modes of clinical presentations. The reported symptoms of a symptomatic proximal tibiofibular synostosis include pain at the proximal tibiofibular junction [5], pain in the ipsilateral ankle [10], and restricted range of motion in the ipsilateral ankle [8], angular deformity [11] and leg length discrepancy [12]. Asymptomatic tibiofibular synostosis does not require any specific treatment. For those causing ankle disabilities, ankle pain or cosmetic defect, corrective osteotomy or resection of the bony bridge following maturation of the callus is advised. The best recommended treatment is conservative but surgery may be advised for athletically active or sports-person [13]. cases with types 1 and 2 were considered to occur before the growth spurt and those with type 3 were considered to occur after the growth spurt. Nishikawa et al [7] also presented another case of Type 4 synostosis.

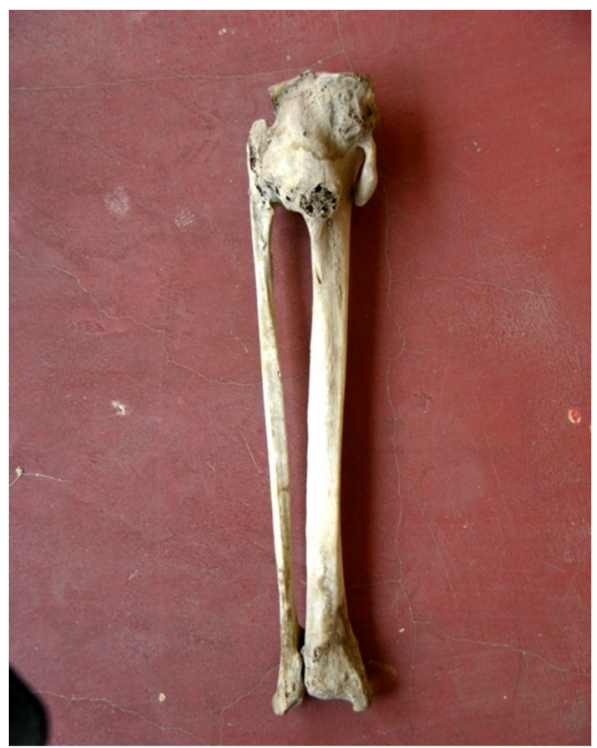

Fig 2: Posterior view of left tibia and fibula showing synostosis

\section{Conclusion}

The author reports another case of Type 4 synostosis. Proximal Tibiofibular synostosis is an extremely rare condition that has been reported only a few times in the literature with varying presentations. Knowledge of this case is useful for the anatomists, radiologists and Orthopaedic surgeons who are dealing with this region.

\section{Funding: Nil}

\section{Conflict of interest: Nil}

\section{Permission from IRB: Yes}

\section{References}

1. Esenkaya I, Unay K. Proximal Tibiofibular Synostosis, an Unexpected Complication of a Proximal Tibial Osteotomy. International Journal of Case Reports in Medicine. 2013; 2013:1-6.

2. Umesan KG. An abnormal bony union between leg bones. International Journal of Case Reports and Images. 2013; 4(6):334-336.

3. Rahm H. Die Tibiofibulare Synostose. Zeitschrift Orthop Chir. 1924; 43:64-69.

4. Jiang-Hue Fu, Chyi-Chyuan Hwang, Tai-Hung Chao. Tibiofibular synostosis in a military soldier. J Med Sci. 2003; 23(2):135-138. 
5. O'Dwyer KJ. Proximal tibio-fibular synostosis. A rare congenital anomaly. Acta Orthopaedica Belgica. 1991; 57(2):204-8.

6. Takai S, Yoshino N, Hirasawa Y. Unusual proximal tibiofibular synostosis. International Orthopaedics. 1999; 23:363-365.

7. Nishikawa T, Yamamoto T, Yoshiya S, Kurosaka M. Intermittent peroneal mononeuropathy due to proximal tibiofibular synostosis. Journal of Neurology. 2003; 250(3):352-353

8. Nishimura T, Nii E, Urawa M, Nishiyama M, Taki S, Uchida A. Proximal Tibiofibular synostosis with 49xxxxy syndrome, a rare congenital bone anomaly. Journal of Orthopaedic Science. 2008; 13(4):390-395.
9. Gamble JG. Proximal tibiofibular synostosis. Journal of Paediatric Orthopaedics. 1984; 4 (2): 243-245.

10. Leninbabu V, Shenbaga N, Komarasamy B, Paul A. Proximal tibiofibular synostosis as a source of ankle pain: a case report. The Iowa Orthopaedic Journal. 2006; 26: 127-129.

11. Solomon L. Bone Growth in Diaphysial Aclasis. The Journal of Bone \& Joint Surgery. 1961; 43B:700-716.

12. Wong K, Weiner DS. Proximal Tibiofibular Synostosis. Clinical Orthopaedics \& Related Research. 1978; 135:45-47.

13. Aneja PS, Aneja M, Sood KS, Asthana S. Bilaterally symmetrical tibiofibular synostosis - A rare incidental finding. International Journal of Biomedical Research. 2013; 04 (06): 292-295.

\section{How to cite this article?}

Tiwari S, Roopashree R, Padmavathi G, Sangeeta M. Proximal Tibiofibular Synostosis and its Clinical Significance: A Case Report. Int J Med Res Rev 2014; 2(2):163-165. doi:10.17511/ijmrr.2014.i02.18. 